\title{
Detection and Identification of Transient Power Quality Disturbance
}

\author{
Xiaohong $\mathrm{Hao}^{1}$, Juan $\mathrm{Cao}^{1, \mathrm{a}}$, Yufang $\mathrm{Han}^{1}$,Qun $\mathrm{Gu}^{1}$
}

\author{
School of Computer and Communication, Lanzhou University of Technology, Lanzhou 730050, \\ China \\ aangelhoodcj@163.com
}

Keywords: Disturbance signals, Wavelet transform, Generalized S transform, Rank-WSVM

\begin{abstract}
Aiming at insufficient classification performance and long classification time in detection and identification of transient power quality disturbance signal, this paper proposed a detection method that uses wavelet transform combined generalized S transform and an identification method named rank wavelet support vector machine(Rank-WSVM) which uses the complex Gaussian wavelet kernel function based on the original method for the detection and identification. Firstly, high and low frequency components are obtained by wavelet transform .then, the low frequency components were selected to through generalized S transform and extract feature vector. Finally achieving signal classification through Rank-WSVM which using complex Gaussian wavelet kernel function. Simulation results show that, the proposed method can improve the classification accuracy and can reduce the classification time.
\end{abstract}

\section{Introduction}

The classification of power quality disturbance usually extracts the perturbation feature and then classifies it by a classifier. In terms of feature amount extraction, wavelet transform and S transform are the common extraction means. Wavelet transform has the characteristics of multi-scale analysis. In [1-2], wavelet packet decomposition is used to extract wavelet packet energy as eigenvalue to classify several common perturbations. S transform has good time-frequency characteristics and can be performed by extracting a single frequency information of signal analysis. In [3-4], the S-transform is used to extract the fundamental energy of the signal and the energy of the 3rd, 5th and 7th harmonics as the feature quantity, but its ability is not prominent when resolving the complex perturbation with harmonic. In [5], the Gaussian window adjustment factor is introduced to improve the traditional S-transform which enhance the ability of the traditional S-transform to distinguish harmonic disturbance signals. However, there is a problem that the S-matrix is large and the computation time is long. In terms of disturbance identification, the concept of multi-label classification has been introduced into the classification of power quality compound disturbance in [6]. In [7], the wavelet-based support vector machine (Rank-WSVM) is proposed and applied to the combined power quality classification. But Gauss wavelet and Morlet wavelet are chosen to replace the original support vector kernel. In [8], it is proved that SVM based on complex Gaussian wavelet kernel function is superior to ordinary Gaussian kernel function and scale kernel function.

Based on previous studies, this paper proposes a detection method based on wavelet transform combined with generalized S-transform and an identification method named Rank-WSVM which uses the complex Gaussian wavelet kernel function. Firstly, the high and low frequency components are obtained by wavelet transform, then the generalized S transform is used to extract the eigenvectors, and the signals are classified by Rank-WSVM using complex Gaussian wavelet kernel function. 


\section{The detection method that uses wavelet transform combined generalized S transform}

\section{1 wavelet transform and the generalized $S$ transform}

Wavelet transform is developed based on the short-time Fourier transform, which is a time-frequency analysis method. The continuous wavelet transform of $f(t)$ is defined as

$$
W T_{f}(a, b)=|a|^{-1 / 2} \int_{-\infty}^{\infty} f(t) \Psi\left(\frac{t-b}{a}\right) d t
$$

Where $a$ is the translation factor, $a \neq 0 ; b$ is the scaling factor; and $\psi$ is the wavelet mother function.

In the process of frequency characteristic extraction of disturbance signal, when frequency of two kinds of disturbance signals are similar, frequency overlapping will occur when S-transform two-dimensional contour is used to identify frequency characteristics, which is not good for frequency feature extraction. In order to improve the time-frequency resolution of the signal, we can introduce $\alpha, \beta$ in the S-transform. Therefore, the generalized S-transform can be defined as

$$
S(\tau, f)=\int_{-\infty}^{\infty} h(t) \frac{\alpha|f|^{\beta}}{\sqrt{2 \pi}} e^{-\frac{\alpha^{2}(t-\tau)^{2} f^{2}}{2 a^{2}}} e^{-j 2 \pi f t} d t
$$

Where $\alpha$ is the Gaussian window amplitude factor, $\beta$ is the frequency scale stretching factor. If and only if $\alpha=1, \beta=1$, the generalized $S$ transform is equal to $S$ transform; When $\alpha>1$ or $\beta>1$, the time resolution of the signal increases and the frequency resolution decreases; When $\alpha<1$ or $\beta<1$, the frequency resolution increases and the time resolution decreases.

\subsection{The detection method that uses wavelet transform combined generalized $\mathrm{S}$ transform}

In the study, the sampling frequency is $2000 \mathrm{~Hz}$, and the range of the high frequency component after wavelet transform is $500 \sim 1000 \mathrm{~Hz}$ (the oscillation frequency is usually concentrated in this frequency band). The range of the low frequency component is $0 \sim 500 \mathrm{~Hz}(3,5$ and 7 Harmonics are in this band). In this paper, we choose the following eigenvalues to form eigenvectors:

The sum of the amplitude squares of the high frequency components after wavelet transform;

Fundamental component and the sum of the amplitude squares of the 3rd, 5th and 7th harmonic components;

Maximum and minimum amplitude of fundamental component;

Signs of interrupt signal: The amplitude of the fundamental signal is 0.1 times than the amplitude of the normal signal continuously;

Signs of sag signal: The amplitude of the fundamental signal is $0.1 \sim 0.9$ times than the amplitude of the normal signal continuously;

Signs of swell signal: The amplitude of the fundamental signal is 1.1 times the amplitude of the normal signal continuously.

First, sampling the signal and determining the adjustment factors $\alpha$ and $\beta$. Then, decomposing the signal into high and low frequency components through wavelet transform. The high frequency components are $500 \sim 1000 \mathrm{~Hz}$, which are used to distinguish the characteristic signals of oscillatory signals. Extracting the amplitude information of the fundamental wave and the 3, 5 and 7 th harmonics after making S-transform for low-frequency components. According to the amplitude information of the fundamental wave to judge whether there are interrupt, swell and sag signals. According to the amplitude information of harmonic to determine whether it contains the sub-harmonic.

\section{The identification method named Rank-WSVM with the complex Gaussian wavelet kernel function}

\subsection{Multi-label classification based on ranking method}

At present, most of the schemes to solve the problem of multi-label classification are qualitative classification. Generally, the multi-label classification constructs a binary real function 
$f: \chi \times \psi \rightarrow R$ based on qualitative classification during the training process. For the samples $x_{i} \in \chi$, all the labels are ranked according to the value of $f\left(x_{i}, \cdot\right)$, and the top-ranking labels labeled $x_{i}$ are more likely to have labels. The more successful classifier should make all label values $Y_{i}$ that belong to are bigger than all labels not belonging to $Y_{i}$, that is to say, for all $y_{1} \in Y_{i}$ and $y_{2} \notin Y_{i}$, $f\left(x_{i}, y_{1}\right)>f\left(x_{i}, y_{2}\right)$. Define a sorting function $f_{\text {rank }}(\cdot, \cdot)$ by $f(\cdot, \cdot)$, as a result, when $f\left(x_{i}, y_{1}\right)>f\left(x_{i}, y_{2}\right), f_{\text {rank }}\left(x_{i}, y_{1}\right)>f_{\text {rank }}\left(x_{i}, y_{2}\right)$. Through $f(\cdot, \cdot)$, it is easy to get the corresponding display multi-tag classifier the $h(\cdot)$, and define $h\left(x_{i}\right)=\left\{y \mid f\left(x_{i}, y\right)>t\left(x_{i}\right), y \in \psi\right\}$, which $t(\cdot)$ is a threshold function.

In order to better distinguish the effect of different multi-label classification differences, making the evaluation of the classifier has good balance and comprehensiveness, set the test sample set as $T=\left\{\left(x_{1}, Y_{1}\right),\left(x_{2}, Y_{2}\right), \ldots,\left(x_{n}, Y_{n}\right)\right\}$, and the relevant literatures define the following evaluation indicators: Hamming loss, one- error, Ranking loss, Coverage, Average precision. Among five evaluation indexes, the average precision is higher, the other four evaluation indexes are smaller, and the classification performance is better. The Hamming loss is related to the multi-label classifier $h(\cdot)$, while the other four evaluation indices are related to the ranking real function $f(\cdot, \cdot)$.

\subsection{Complex Gaussian Wavelet Kernel Functions}

Definition $1 \exists \psi(x) \neq 0, \psi(x)$ is a complex Gaussian wavelet

$$
\psi(x)=C_{P} \exp (-i x) \exp \left(-x^{2}\right)
$$

Definition 2 Complex Gaussian wavelet kernel function

$$
\left\{\begin{array}{l}
K\left(x, x^{\prime}\right)=\prod_{i=1}^{n} \psi\left(\frac{x_{i}-b_{i}}{a_{i}}\right) \cdot \psi\left(\frac{x_{i}^{\prime}-b_{i}^{\prime}}{a_{i}^{\prime}}\right) \\
\text { s.t. } \quad x, x^{\prime} \in R^{n} \\
a_{i}, a_{i}^{\prime}, b_{i}, b_{i}^{\prime} \in R ; a_{i} \neq 0, a_{i}^{\prime} \neq 0
\end{array}\right.
$$

Where, $a_{i}$ and $a_{i}^{\prime}$ are the expansion factor, while $b_{i}$ and $b_{i}^{\prime}$ are the displacement factor.

\subsection{Constructing Rank-WSVM based on the complex Gaussian wavelet kernel}

The goal of the Rank-SVM is to maintain a maximum interval value and to have a minimum ranking loss at the same time. Therefore, we can get the final description of the optimization problem by approximating the Heaviside function of linearized constraint:

$$
\left\{\begin{array}{l}
\min _{w_{j}, j=1, \cdots,} \frac{1}{2} \sum_{k=1}^{Q}\left\|w_{k}\right\|^{2}+C \sum_{i=1}^{m} \frac{1}{\left|Y_{i} \| \bar{Y}_{i}\right|} \sum_{(k, l) \in Y_{i} \times \bar{Y}_{i}} \xi_{i k l} \\
<w_{k}-w_{l}, x>+b_{k}-b_{l} \geq 1-\xi_{i k l}, \\
(k, l) \in Y_{i} \times \bar{Y}_{i}, \xi_{i k l} \geq 1, C>0
\end{array}\right.
$$

Where $C$ is the penalty factor, $\langle\cdot, \cdot\rangle$ is the vector dot product, $w$ is the coefficient vector of the classified hyperplane in the feature space, $b$ is the threshold of the classification surface, $k$ is the effective label set of $x$, and $l$ is the invalid label set. Among them, the first part reflects the complexity of the algorithm, the second part is the experience Hamming loss.

To solve the above optimization problem, the key is to obtain the estimated value $w_{j}^{*}, b_{j}^{*}(j=1,2, \cdots Q)$ of parameter $w_{j}, b_{j}$. In the process of classifying test data, $\left\langle w_{k}, x\right\rangle+b_{k}$ value of each label should be calculated and be used as ranking function value, and then ranking the label according to ranking function value.

The choice of the kernel function of SVM determines the structure of the feature space and directly affects the complexity and precision of the algorithm. Wavelet Support Vector Machine 
(SVM) is a combination of wavelet technology and support vector machine (SVM), which utilizes the high frequency subdivision property of wavelet function to find the best approximation or classification in multi-dimensional wavelet or the spaces of wavelet kernel. In this paper, a complex-Gaussian wavelet kernel is used to replace the kernel function in Rank-SVM, and a Rank-WSVM algorithm based on complex Gaussian wavelet kernel is proposed.

\section{The Specific implementation steps of the Classification algorithm}

The Specific implementation steps of the Classification algorithm is:

1) The S-transform matrix is obtained by making wavelet transform and generalized S-transform for the original signal.

2) Extracting the eigenvectors and dividing them into training samples and test samples.

3) Classifying the data by the Rank-WSVM algorithm that uses complex Gaussian wavelet kernel.

4) Obtaining and evaluating the classification results.

Algorithm flow chart shown in Figure 1:

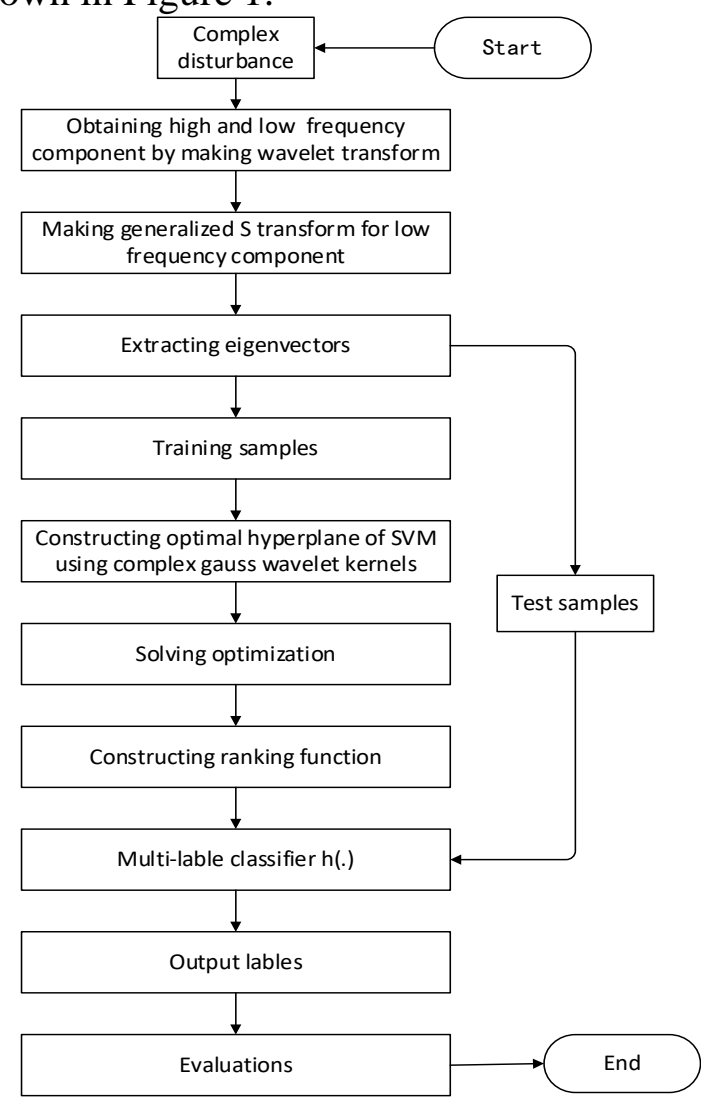

Fig. 1 Flow chart of algorithm

\section{Simulation}

Generating harmonic signals, voltage interruption, voltage sag and voltage swell, electromagnetic oscillation and its double, triple complex disturbance signals randomly. Signal sampling frequency $f_{s}=2 \mathrm{kHz}$, the sampling interval is $0.5 \mathrm{~ms}$, the number of sampling points is 1000 , the fundamental signal frequency is $50 \mathrm{~Hz}$. Each type of disturbance randomly generated 200 samples, One hundred samples were randomly selected as training samples, and another 100 samples were used as test samples.

Taking a single voltage sag signal as an example, the disturbance signal is simulated and analyzed, and the sampling signal is shown in Fig 2. The high and low frequency component of the signal will be obtained by making the single-scale 1-D wavelet transform for the signal and the high 
and low frequency component is shown in Fig 3. It can be seen from the high frequency components in the figure, this signal has no voltage oscillation disturbance. Then making generalized S-transform for low frequency components and extracting amplitude information when the frequency is $50,150,250$ and $350 \mathrm{~Hz}$.

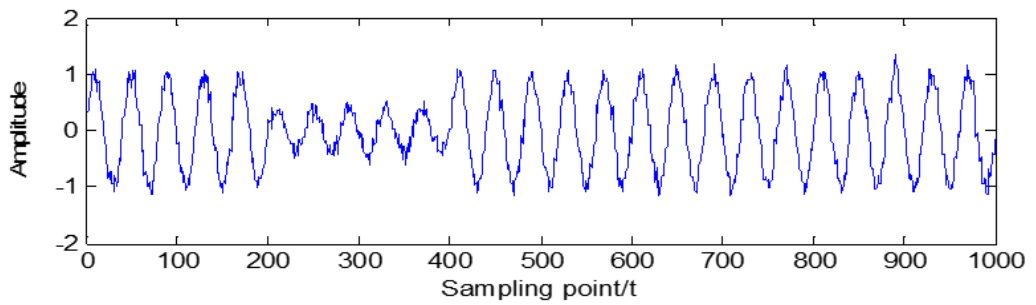

Fig. 2 Voltage sag signal with 20dB white noise

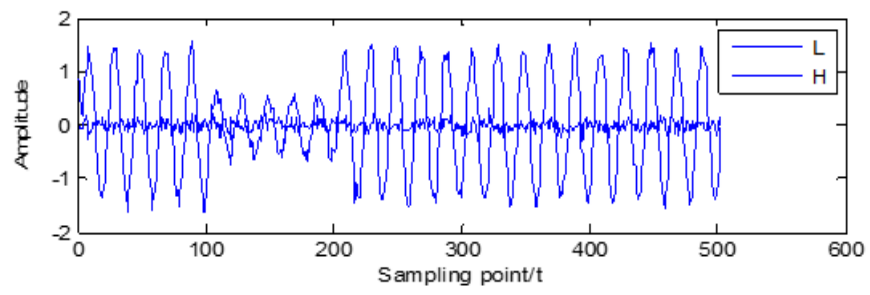

Fig. 3 High and low frequency components after wavelet transform

It can be seen that there is no harmonic disturbance signal by analyzing the amplitude information when the frequency is $50,150,250$ and $350 \mathrm{~Hz}$.

From Fig. 2 to Fig. 3, the specific eigenvector values can be obtained and be showed in Table 1.

Table 1 Feature values of signal involving voltage sag

\begin{tabular}{|c|c|c|}
\hline Number & Eigenvectors & Feature values \\
\hline 1 & Amplitude sum of squares of High & 0.495 \\
\hline 2 & $\begin{array}{l}\text { Frequency component } \\
\text { Amplitude sum of squares of 3rd } \\
\text { harmonic }\end{array}$ & 0.352 \\
\hline 3 & $\begin{array}{l}\text { Amplitude sum of squares of } 5 \text { th } \\
\text { harmonic }\end{array}$ & 0.267 \\
\hline 4 & $\begin{array}{l}\text { Amplitude sum of squares of } 7 \text { th } \\
\text { harmonic }\end{array}$ & 0.395 \\
\hline 5 & $\begin{array}{l}\text { Amplitude sum of squares of } \\
\text { Fundamental component }\end{array}$ & 370.931 \\
\hline 6 & Signs of interrupt signal & 0 \\
\hline 7 & Signs of sag signal & 1.000 \\
\hline 8 & Signs of swell signal & 0 \\
\hline 9 & $\begin{array}{l}\text { The maximum amplitude of the } \\
\text { fundamental component }\end{array}$ & 0.681 \\
\hline 10 & $\begin{array}{l}\text { The minimum amplitude of the } \\
\text { fundamental component }\end{array}$ & 0.274 \\
\hline
\end{tabular}

In order to verify the effectiveness of the proposed detection algorithm, the detection method that combining wavelet transform and generalized S-transform is compared with common S-transform and generalized S-transform under the same noise condition. Specific classification results is showed in Table 2.From Table 1 we can see, the traditional S-transform and the generalized S-transform without combining wavelet transform have more than $97 \%$ accuracy . However, in the time of collecting and training sample, the detection method that uses wavelet transform combined with generalized S-transform have obvious advantages, only is $1 / 3$ compared to the traditional detection methods. 
Table 2 statics results of three sorting methods

\begin{tabular}{cccc}
\hline $\begin{array}{c}\text { Classification } \\
\text { method }\end{array}$ & S Transform & $\begin{array}{c}\text { Generalized S } \\
\text { transform }\end{array}$ & $\begin{array}{c}\text { Wavelet+Generalized } \\
\text { S }\end{array}$ \\
\hline transform
\end{tabular}

In order to verify the effectiveness of the proposed recognition algorithm, the classification performance of Rank-WSVM with Gaussian wavelet kernel function is compared with Rank-WSVM with standard Gauss kernel function and scale kernel function under different noise conditions. Specific classification results is showed in Table 3.It can be seen from Table 3, Rank-WSVM method with Gaussian wavelet kernel function which is proposed in this paper has a significant improvement in each evaluation index. This shows that the recognition performance of the algorithm which is proposed in this paper is superior to the common Rank-WSVM method with Gaussian kernel function and scale kernel function. And the result of classification has no obvious difference under different noise levels, which shows that the method is not sensitive to noise and has good noise resistance.

Table 3 Performance comparison of Rank-WSVM with different wavelet kernel

\begin{tabular}{lcccccc}
\hline $\begin{array}{c}\text { Rank-WSVM with different kernel } \\
\text { function }\end{array}$ & $\begin{array}{l}\text { SN } \\
\text { /dB }\end{array}$ & $\begin{array}{c}\text { Hamming } \\
\text { loss }\end{array}$ & $\begin{array}{c}\text { Ranking } \\
\text { loss }\end{array}$ & $\begin{array}{c}\text { One } \\
\text { Error }\end{array}$ & $\begin{array}{c}\text { Cover } \\
\text { age }\end{array}$ & $\begin{array}{c}\text { Average } \\
\text { Precision }\end{array}$ \\
\hline Complex Gaussian Wavelet Kernel & & $\mathbf{0 . 1 5 1}$ & $\mathbf{0 . 0 7 1}$ & 0.072 & $\mathbf{2 . 0 0 9}$ & $\mathbf{0 . 9 1 2}$ \\
Gaussian Wavelet Kernel & 20 & 0.171 & 0.080 & 0.074 & 2.156 & 0.906 \\
Morlet Wavelet Kernel & & 0.152 & 0.074 & $\mathbf{0 . 0 6 8}$ & 2.105 & 0.908 \\
\hline Complex Gaussian Wavelet Kernel & & $\mathbf{0 . 1 3 1}$ & $\mathbf{0 . 0 5 8}$ & $\mathbf{0 . 0 5 4}$ & $\mathbf{1 . 9 9 8}$ & $\mathbf{0 . 9 3 0}$ \\
Gaussian Wavelet Kernel & \multirow{2}{*}{30} & 0.168 & 0.064 & 0.056 & $\mathbf{1 . 9 9 8}$ & 0.926 \\
Morlet Wavelet Kernel & & 0.133 & 0.063 & 0.056 & 2.010 & 0.927 \\
\hline Complex Gaussian Wavelet Kernel & & $\mathbf{0 . 1 3 5}$ & $\mathbf{0 . 0 6 2}$ & $\mathbf{0 . 0 4 9}$ & $\mathbf{2 . 0 0 1}$ & $\mathbf{0 . 9 2 8}$ \\
Gaussian Wavelet Kernel & 40 & 0.143 & 0.078 & 0.076 & 2.107 & 0.917 \\
Morlet Wavelet Kernel & & 0.137 & 0.063 & 0.051 & 2.009 & 0.926 \\
\hline Complex Gaussian Wavelet Kernel & & $\mathbf{0 . 1 3 7}$ & $\mathbf{0 . 0 6 8}$ & $\mathbf{0 . 0 6 2}$ & $\mathbf{2 . 0 5 1}$ & $\mathbf{0 . 9 2 0}$ \\
Gaussian Wavelet Kernel & 50 & 0.159 & 0.088 & 0.071 & 2.056 & 0.913 \\
Morlet Wavelet Kernel & & 0.139 & 0.070 & $\mathbf{0 . 0 6 2}$ & 2.054 & 0.918 \\
\hline
\end{tabular}

\section{Conclusions}

In this paper, a detection method that uses wavelet transform combined generalized S transform and an identification method named rank wavelet support vector machine(Rank-WSVM) which uses the complex Gaussian wavelet kernel function are applied to the classification of composite power quality. Firstly, high and low frequency components are obtained by making wavelet transform for the original signal then, the low frequency components were selected to through generalized $S$ transform and extract feature vector .finally achieving signal classification through Rank-WSVM which using complex Gaussian wavelet kernel function. Simulation results show that, the proposed method can improve the classification accuracy and can reduce the classification time. The method proposed in this paper can achieve high accuracy of classification and good noise immunity based on full consideration of diversity of power quality disturbance signals, and the classification time is far less than that uses traditional algorithms.

\section{References}

[1] Gaing Z L. Wavelet-based PNN for power disturbance recognition and classification [J]. IEEE Transactions on Power Delivery, 2004, 19(4): 1560-1568. 
[2] He Chaohui, Huang Chun, Liu Bin, et al. Power quality disturbances classification based on kernel principal component analysis of wavelet coefficients and probabilistic neural networks [J]. Proceedings of the CSU-EPSA, 2010, 22(2): 77-81.(in Chinese)

[3] Reddya M J B, Raghupathya R K, Venkatesha K P. Power quality analysis using discrete orthogonal S-transform ( DOST) [J]. Digital Signal Processing, 2012, 9(13): 1-6.

[4] Tang Qiu, Wang Yaonan, Guo Siyu, et al. Power quality disturbance detection base on transform and PNN[J]. Chinese Journal of Scientific Instrument, 2009, 30(8): 1668-1673.(in Chinese)

[5] Xu Fangwei, Yang Honggeng, Ye Maoqing. Classification for power quality short duration disturbances based on generalize S-transform[J]. Proceedings of the CSEE, 2012, 32(4): 77-84.(in Chinese)

[6] Zhou Luowei, Guan Chun, Lu Weiguo. Application of multi-label classification method to catagorization of multiple power quality disturbances[J]. Proceedings of the CSEE, 2011, 31(4): 45-50(in Chinese).

[7] Zhang Qiaoge, Liu Zhigang, Zhu Ling, Zhang Yang. Classification of Composite Power Quality Disturbances Based on Multilable Rank-WSVM [J]. Proceedings of the Chinese Society for Electrical Engineering, 2013, 33(28): 114-120.

[8] Chen Zhongjie, Cai Yong, Jiang Gang. Research on support vector machine for Complex Gaussian Wavelet Kernel [J] .Application Research of Computers,2012, 29(9): 3263-3265. 